\title{
TOWARDS OPTIMAL IMAGING AND MICROANALYSIS IN VARIABLE PRESSURE AND LOW VOLTAGE SEM
}

\author{
Brendan J Griffin
}

Centre for Microscopy and Microanalysis, The University of Western Australia, 35 Stirling Highway, Crawley, WA 6009

The parameters: accelerating voltage, primary electron beam current, working distance, gas pressure, gas type, detector bias, and detector design have been measured in terms of their influence on contrast in "secondary electron" imaging of a conductive sample in variable pressure SEM.

There were two principal aims of the initial phase of this project: a) the characterisation of the influence of these parameters, and b) to identify optimal imaging conditions for a "new generation (2003)" LEO 1555 FEG VPSEM and those for a "first generation (1990)" ElectroScan E-3 (tungsten filament) ESEM. The images were collected from a silver sphere mounted on a carbon tab. For comparative purposes, the average intensities of $100 \times 100$ pixel regions were measured, using Adobe Photoshop, from "flat" regions on the top of the sphere and from the adjacent carbon. Images were 8-bit and the brightness and contrasts were adjusted for each measurement series so that all images within a data set were within the digital bounds (0-255).

The ESEM data set for these variables are coherent and in absolute terms show typical amplification curves with pronounced maxima (figure 1). When plotted as a signal ratio $(\mathrm{Ag}: \mathrm{C})$ the data show different trends. Variations in detector bias and working distance give linear changes in the contrast ratio whereas the effect of accelerating voltage is a power function and gas pressure is a typical gas amplification curve. Notably the maximum of the contrast ratio for variation in gas pressure occurs at a different pressure to those for the individual contrast data.

The second phase of this project was to evaluate the potential of using an elemental L:K x-ray ratio as an indicator of charging rather than the Duane-Hunt Limit (DHL). Three reasons initiated this work; (a) the large error associated with the DHL measurement, (b) the inaccessibility of the DHL when using high accelerating voltage (e.g. $30 \mathrm{kV}$ ), and (c) a desire to study time-related charge effects and so the need for quick and accurate measurements.

DTSA model data and initial instrument-based measurements have been completed and indicate that the impact of the overvoltage ratio influence on suitably selected emissions for a particular accelerating voltage can be very high (figure 2). Both model and measured data indicate a sensitivity of $\sim 4$ ev per 0.05 change in $\mathrm{Cu} \mathrm{L}: \mathrm{K}$ peak integral value. This is a significant improvement on direct DHL measurements.

New data from the application of these approaches to both ESEM (ElectroScan E-3 and XL30) and VPSEM (LEO 1555 SUPRA VPSEM) will be presented. Use of other spectral pairs as charge monitors at other accelerating voltages and time-related charge effects will be explored. 

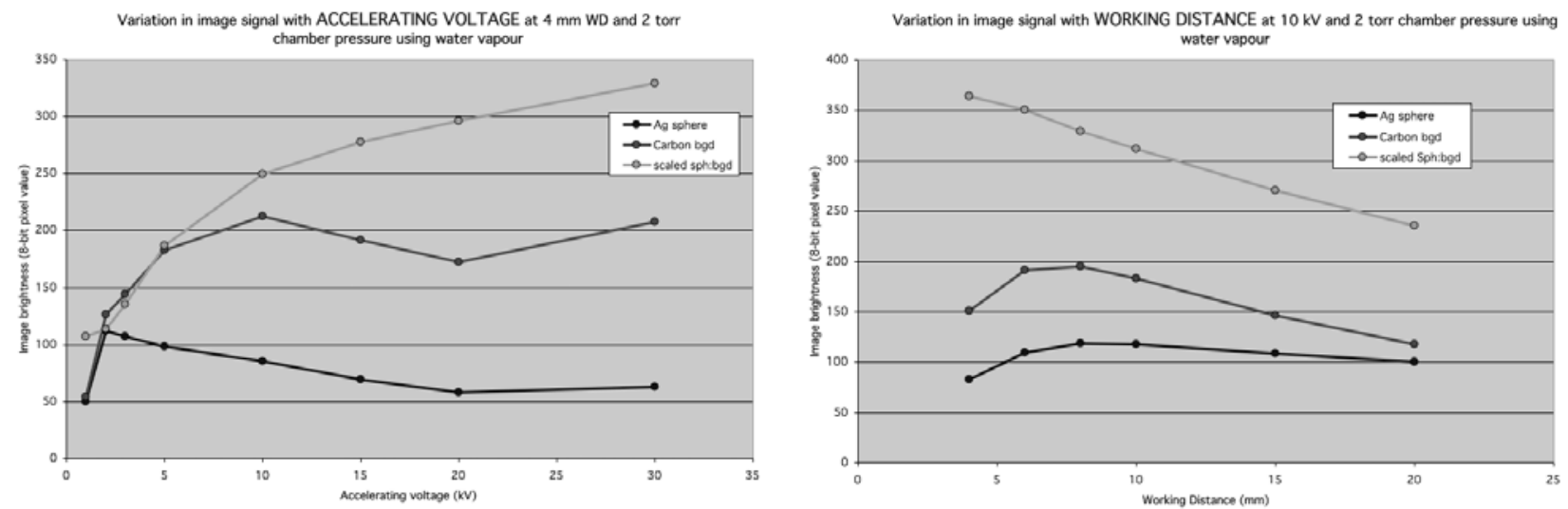

(B)

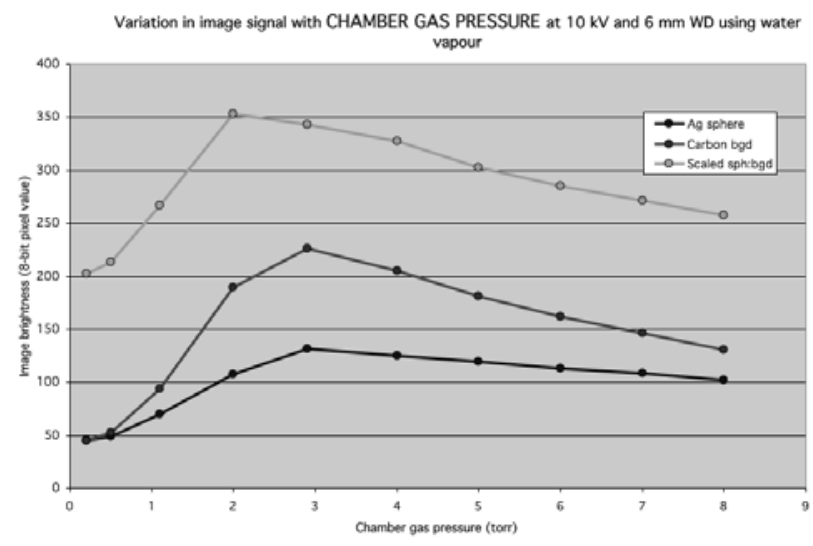

(C)

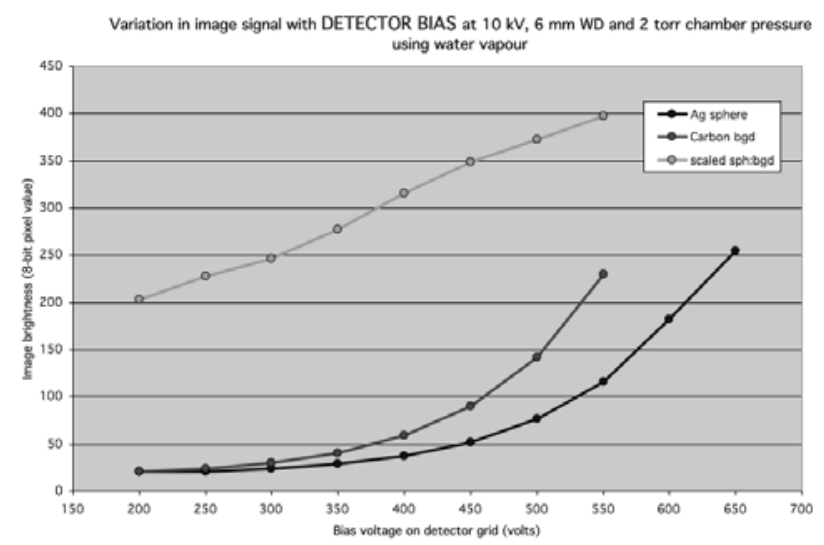

(D)

Figure 1: Image contrast variation with accelerating voltage (A), working distance (B), gas pressure (C), and detector bias (D) under conditions as noted from a silver sphere on a carbon tab background in an ElectroScan E-3 ESEM.

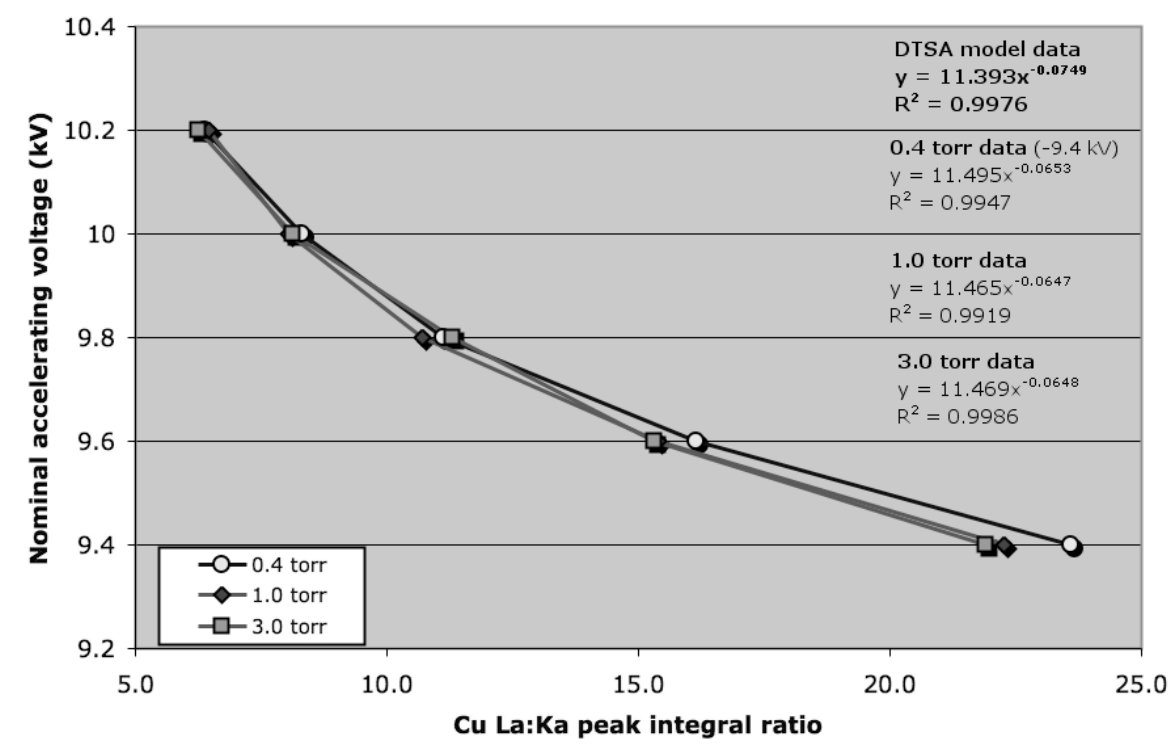

Figure 2: Variation in $\mathrm{Cu} \mathrm{K}: \mathrm{L}$ peak ratio with accelerating voltage and gas pressure from EDS x-ray spectra from a grounded polished $\mathrm{Cu}$ sample in an ElectroScan E-3 ESEM and DTSA model data. 